\title{
The Onset of Normal Field Instability in a Ferrofluid in a Reduced Gravity Environment.
}

\author{
Krull, Brendan; Rundle, Tessa; LeCaptain, Kevin; Barhite, Justin; Gear, Amelia \\ Carthage College Physics Dept.
}

$9 / 14 / 14$

\begin{abstract}
A ferrofluid is a magnetic liquid comprised of nanoscale ferrous particles suspended in a low-viscosity carrier fluid. When subjected to a magnetic field, the surface of a ferrofluid deforms into peaks and valleys along the magnetic field lines. The onset of surface deformation is called the normal field instability. The theory describing the NFI identifies a critical magnetic field below which no magnetically driven surface deformations occur. The critical field depends on the gravitational acceleration and, according to the theory, should disappear as local gravitational acceleration approaches zero. While there have been previous demonstrations of the normal field instability in a reduced gravity environment, data has been inconclusive on the existence of a critical magnetic field in reduced gravity. For our work, we designed a sounding rocket payload for a suborbital rocket mission. Our experiment incorporates a ferrofluid sample and a uniform magnetic field which can be varied across a discrete range of values to observe surface deformations at different applied fields. During the microgravity portion of the rocket's flight, we obtain video of the ferrofluid's behavior and compare it to data taken in Earth's gravity. A team of five undergraduate students designed and built the payload that will characterize the role of gravity in setting the critical magnetic field strength for the onset of the NFI. Data will be used to further understanding of ferrofluid behavior in a microgravity environment to advance its use in various space-based applications.
\end{abstract}

\section{Mission Requirements and Description}

Our objective was to record video of a ferrofluid's response to various external magnetic fields in microgravity and compare it to the response on Earth. Additionally, we expected to find a difference between the critical fields required to induce the deformation in microgravity in Earth's gravity. To do this, at least one video of the ferrofluid's deformation through a series of magnetic field levels in microgravity would have to be collected. A magnetometer would record the magnetic field level being applied at each specific time.

In order to have liquids aboard our flight, our experiment needed to be inside of a secondary containment vessel, to protect the other experiments in case of a leak. The Rocksat team from the previous year had a secondary containment vessel made, so our experiment had to fit inside that space.

Previous work done by Friedrichs and Enge ${ }^{[1]}$ theorizes the effect of gravity on the deformation of ferrofluid in a magnetic field.

We would like to thank Dr. Kevin Crosby for his supervision during the project, as well as the generous support from the Wisconsin Spacegrant Consortium. 
The critical magnetic field for a ferrofluid is shown in equation 1, assuming an infinite plane of ferrofluid with an arbitrary depth $d$ being acted upon by a magnetic field $H$ perpendicular to the ferrofluid plane.

$$
H_{c, \infty}=\sqrt{\frac{(1+\chi)(2+\chi) \sqrt{g \rho \sigma}}{\chi^{2} \mu_{0}}}
$$

The critical magnetic field of the ferrofluid is determined by the magnetic susceptibility $\chi$, ferrofluid density $\rho$, ferrofluid surface tension $\sigma$, the applied gravitational acceleration, and the permeability of free space.

The distinction between the magnetic field $\mathrm{H}$ and the magnetic field $\mathrm{B}$ is that $\mathrm{H}$ is the measurement of magnetic field strength used for magnetic dipoles, and would be used to calculate the force between, say, two bar magnets. $\mathrm{H}$ is relevant in this case, because a ferrofluid is analogous to many tiny interacting bar magnets. B is the magnetic field that would come from a current-carrying wire. In order to create a magnetic field $\mathrm{H}$ inside the ferrofluid, we would need to apply the proper outside magnetic field $\mathrm{B}$. The conversion between these two is shown in equation 2 , which is accurate to within $5 \%{ }^{[1]}$

$$
B=\mu_{0}(1+\chi) H
$$

When the magnetic field $\mathrm{H}$ is above the critical magnetic field, waves begin to form on the ferrofluid's surface. The separation between the peaks of these waves is governed by their wave number $\mathrm{k}$.

$$
k_{c, \infty}=\sqrt{\frac{\rho g}{\sigma}}
$$

Equation 3 describes the wave number for a ferrofluid when the magnetic field $\mathrm{H}$ is equal to $H_{c}$. This is equal to $\frac{2 \pi}{\lambda}$, where $\lambda$ is the peak separation at the critical magnetic field.

Using values of susceptibility and surface tension for our ferrofluid found at $\mathrm{MIT}^{[2]}$, the equations suggest that the critical magnetic field $B$ for the ferrofluid is between 22.8 and $27.1 \mathrm{G}$, and that the peak separation $\lambda$ at the critical magnetic field is between .24 and .33 $\mathrm{mm}$.

According to this equation, during microgravity, the ferrofluid would be expected to form peaks when exposed to any magnetic field. 


\section{Payload Design}

Our payload was designed to record the reaction of a ferrofluid once a constant magnetic field was applied. We decided to use a pair of Helmholtz coils to produce the magnetic field. Helmholtz coils produce a nearly uniform magnetic field between them when they are positioned at a distance equal to their radius away from each other. We decided this was the best option because it would allow for a constant magnetic field while still allowing for clear visibility of the ferrofluid. At the center of the coils we needed a small capsule containing the ferrofluid. After testing the ferrofluid in different containers, we determined that it would be necessary to use a glass container to prevent the ferrofluid from staining the container and making the deformations difficult to see. We then decided to position 2 small cameras on the same plate as the ferrofluid container to record video during the experiment. Initially, we wanted to obtain videos at a side view and a top view, but after deciding on a container with an opaque lid, we decided to put both cameras on the same plate as the ferrofluid and get two views of the capsule.

In order to power the experiment we would house all of the electronics and batteries at the top. The only major design change came after it was discovered that the operational amplifiers could not source enough current to power the coils without overheating itself. We then decided to use relays to control power to the coils.

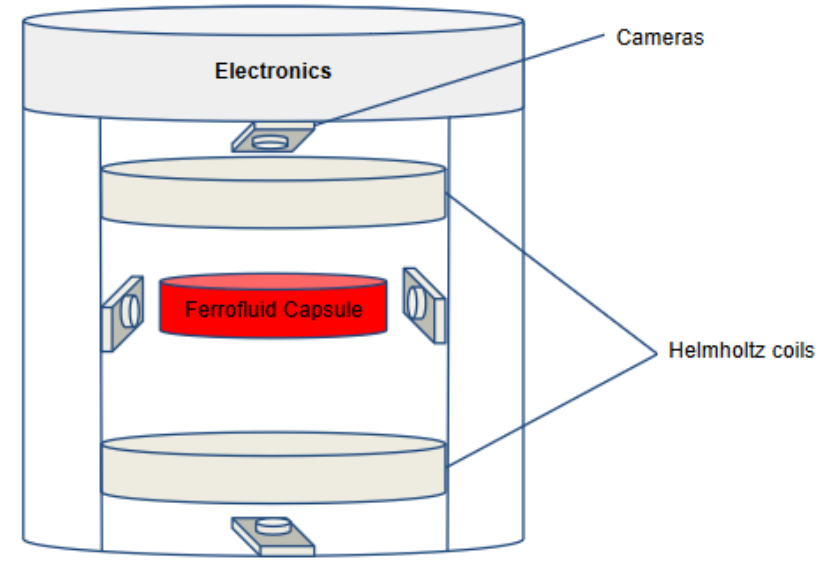

Figure 1. Initial payload design concept. 


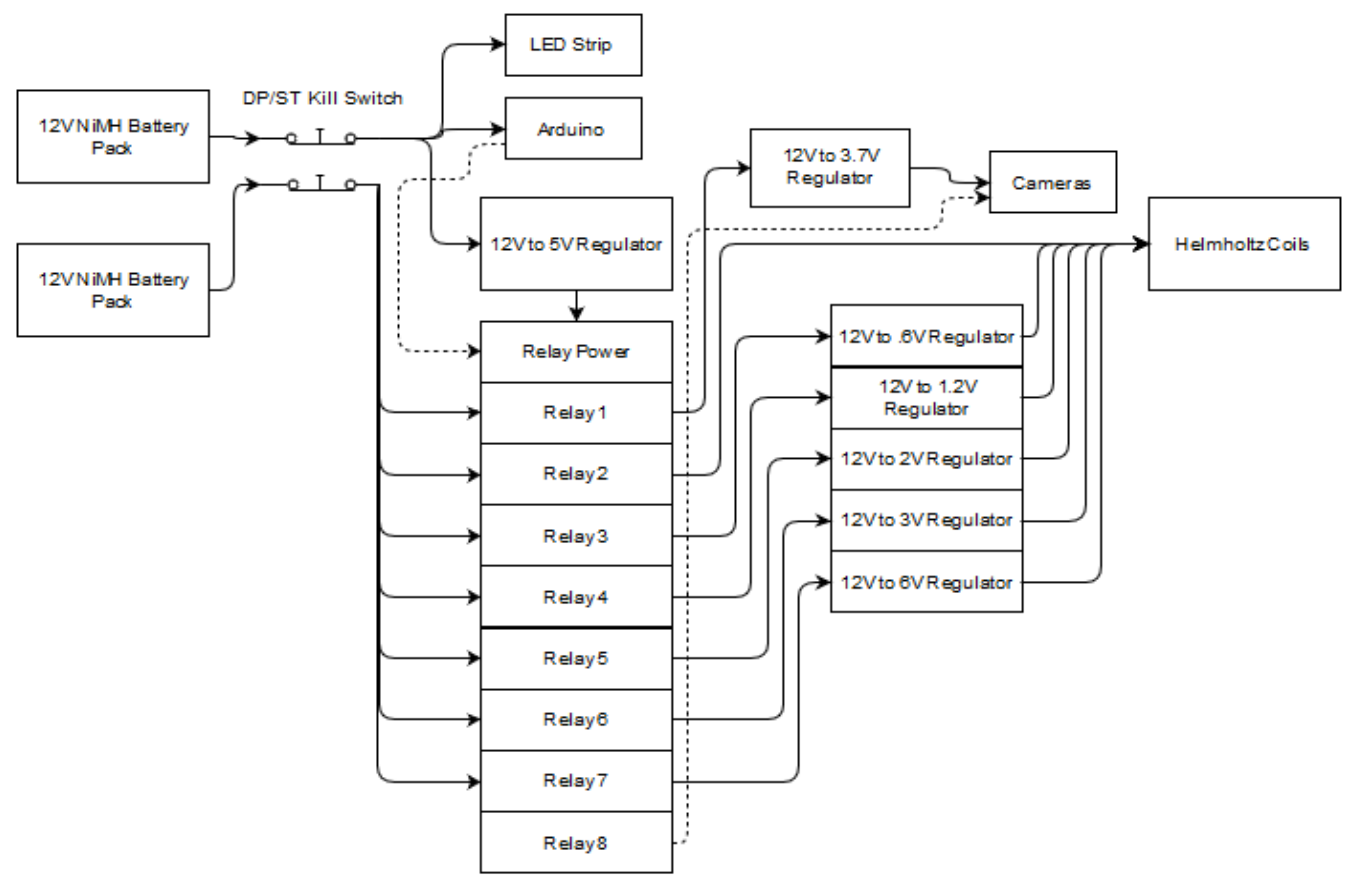

Figure 2: Electronics Block Diagram. Dashed lines show digital signals. Solid lines show power lines.

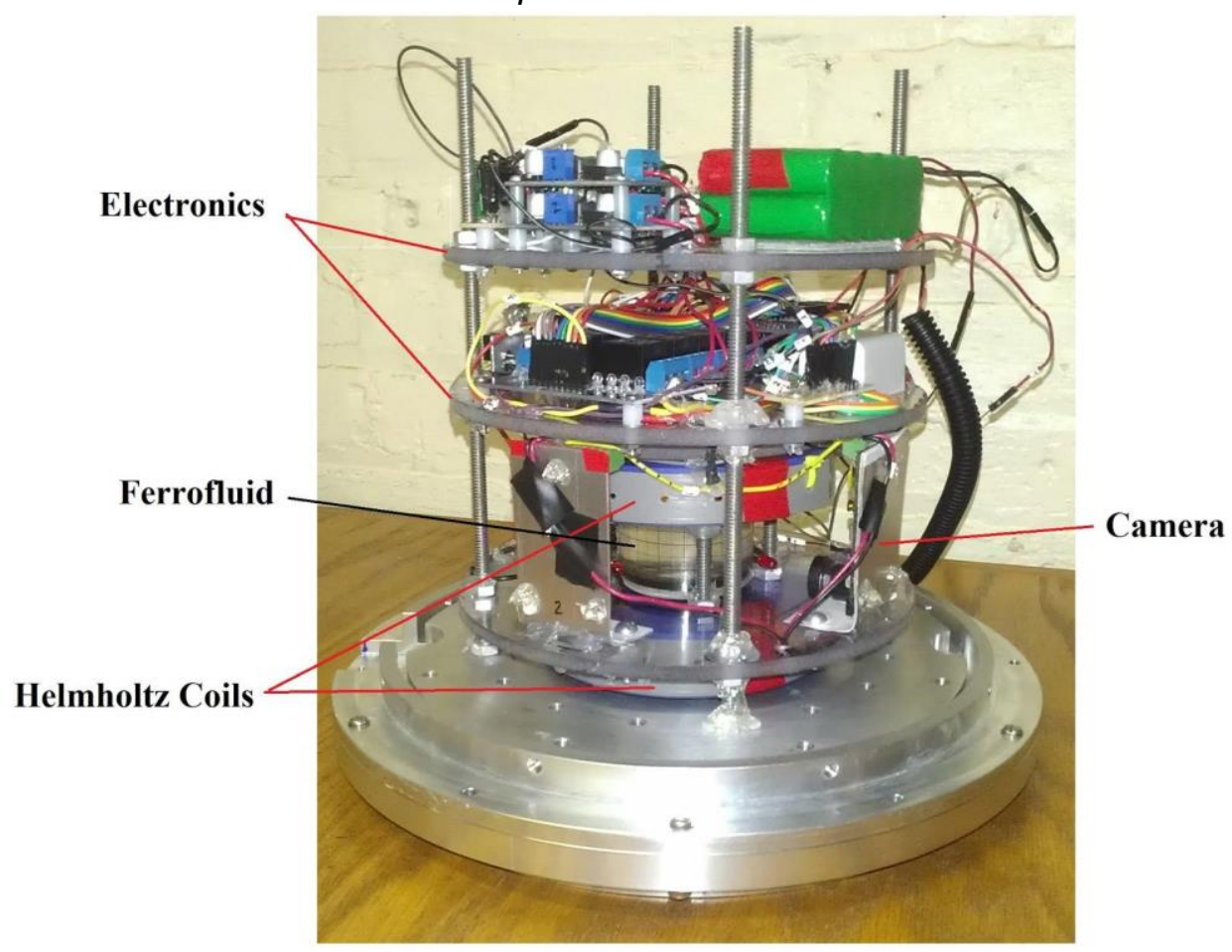

Figure 3: Final payload design. 


\section{Testing Results}

We ran several tests with individual components of our experiment before integrating to ensure that each subsystem would function correctly. Our electrical components (voltage regulators, relays) were tested on their own to ensure they could be used for the purpose we intended. Our Helmholtz coils were tested after construction to verify that heat generated from running the coils for an extended period of time would not affect other electrical components. We tested our magnetometer at different placements, because our magnetometer could only measure magnetic fields between -8 and 8 Gauss and would saturate if placed too close to the magnetic field. We also tested our cameras at several locations in order to have the best possible view of the ferrofluid container. We tested the focus of the cameras to determine the best setting for getting a clear view of the ferrofluid and grid lines on the container. Because the experiment needed to be well lit in order to see the ferrofluid deformation, we ran multiple test runs for different LED placements. The LEDs were placed in the optimal position for lighting the ferrofluid while reducing the amount of glare on the glass container.

Other experiments on the Rocksat-C flight were designed to measure the Earth's magnetic field, which required the installation of steel plates to dissipate the magnetic field in the direction of the other experiment. We determined the magnetic field falloff using one, two, and three steel shielding plates and concluded that the use of three plates would be the best option to minimize the chance of our produced field interfering with another experiment.

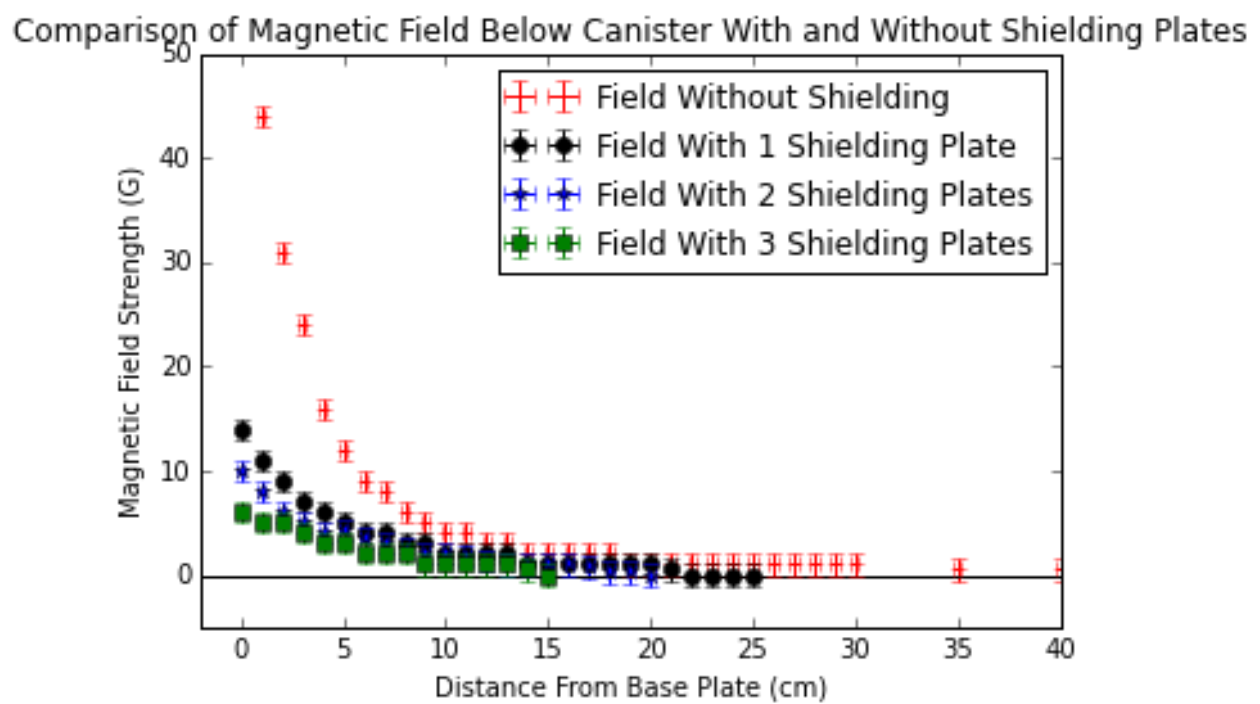

Figure 4: Graph of measured magnetic field below experiment, comparing multiple shielding plates.

After we tested each of our components individually, we ran a series of full mission simulations. With these tests, we ensured that the Arduino was triggering all events at the proper time and the SD cards could read and write correctly. Some simulations were run without secondary containment and the Rocksat-C canister so team members could monitor 
LED status and visually confirm that the simulation was running as planned. We also ran several mission simulations inside secondary containment to obtain video used as ground data.

\section{Mission Results}

Our payload failed to carry out its mission. After de-integration, the experiment was discovered to have turned on without executing the program. Further testing showed that the most likely cause of this failure occurred right at launch. The SD card was most likely jarred by the force of launch, and this caused the Arduino's code to crash. This was an oversight in testing.

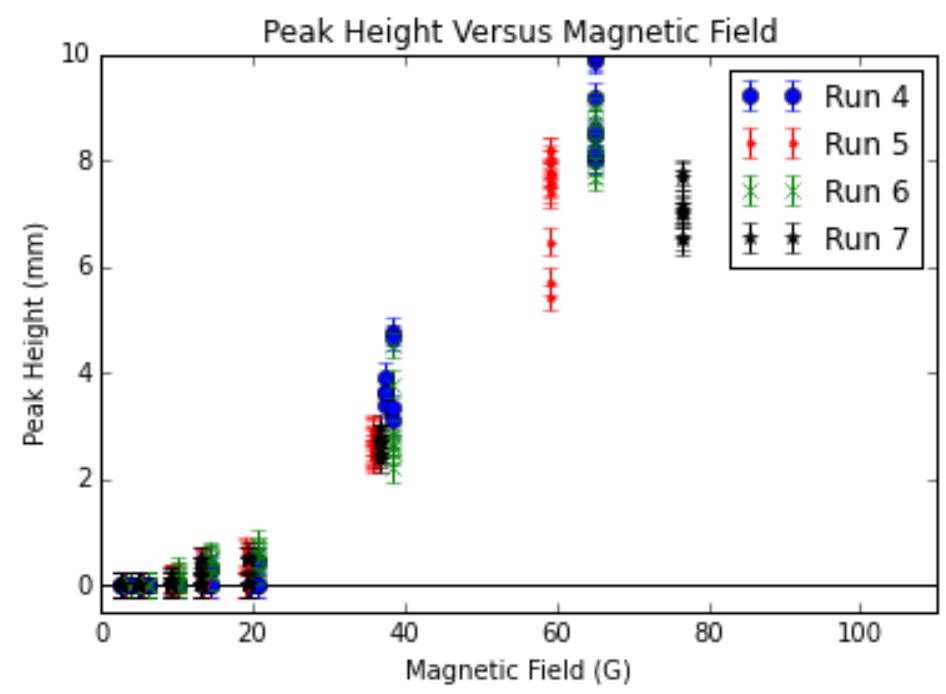

Figure 5: Graph of ferrofluid height vs magnetic field in $1 \mathrm{~g}$

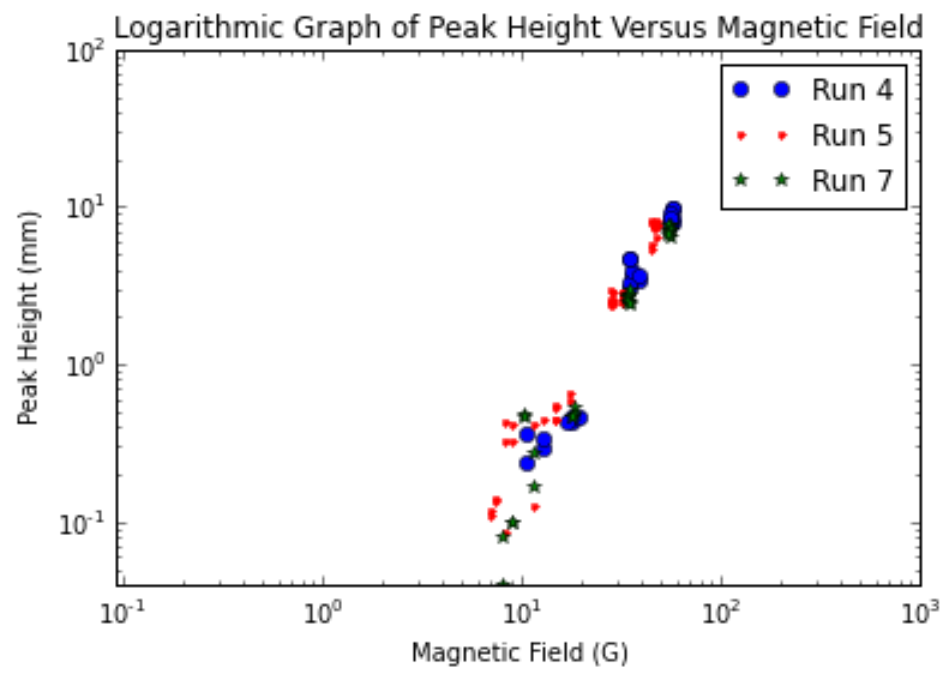


Figure 6: Logarithmic graph of ferrofluid height vs magnetic field in $1 g$

Analysis of the ground data, intended to serve as a comparison point against the zero-g data, revealed that our ferrofluid begins to deform under an applied magnetic field of approximately 15 G. Our calculations predicted that the onset of Normal Field Instability would occur under an applied magnetic field between 22 and $27 \mathrm{G}$ in 1-g, which is close to, but not the same as, our experimental value.

Because we failed to collect zero-g data, we do not have any data to analyze in order to determine the onset on Normal Field Instability in a microgravity environment, and thus cannot determine if the onset occurs under a lower applied magnetic field as predicted.

\section{Conclusions}

Our experiment could use more detail in its ability to select magnetic field levels. An experiment that would build on this one would need to be able to select many more magnetic field levels so that we could obtain more data points near the calculated onset of normal field instability, to determine the accuracy of our calculations and obtain a more precise experimental value.

Because we did not obtain any zero-g data, we are not able to determine the onset of normal field instability in our ferrofluid in microgravity conditions, nor compare our ground data to data obtained in zero-g.

\section{Potential Follow-up Work}

Due to the nature of our payload's failure, it could be run as is on next year's RockSat flight, however, a number of potential improvements could be made.

The ferrofluid container could be improved. Our Helmholtz coils had to be placed farther apart than we would have liked in order to accommodate our container, and a custom container could be made to fit our specifications.

In a future project, it would be very important that a more continuous sample of magnetic field levels could be captured. Since we had to use relays to control the application of magnetic field levels, we had to decide on a very discrete set of magnetic field levels, making it difficult to pinpoint the field level where we first see normal field instability. If we could sweep through a range of magnetic field levels, it would be much easier to pinpoint the onset of normal field instability.

The experiment can be modified to fly on other rockets or on zero-gravity flights.

[1] Friedrichs, Rene, and Andreas Engel. "Pattern and Wave Number Selection in Magnetic Fluids." Physical Review E 64 (2001) 
[2] Amin, Shahrooz, Shihab Elborai, Se-Hee Lee, Xiaowei He, and Markus Zahn. "Surface Tension Measurement Techniques of Magnetic Fluids at an Interface between Different Fluids Using Perpendicular Field Instability." Journal of Applied Physics 97 (2005) 